\title{
Almost topological classification of finite-to- one factor maps between shifts of finite type
}

\author{
ROY ADLER ${ }^{1}$, BRUCE KITCHENS ${ }^{2}$ AND BRIAN MARCUS \\ ${ }^{1,2}$ IBM Research Laboratory, Yorktown Heights, New York 10598, USA: ${ }^{3}$ IBM \\ Research Laboratory, San Jose, California 95193, USA and University of North \\ Carolina, Chapel Hill, North Carolina 27514, USA
}

(Received 12 August 1984)

Abstract. We classify finite-to-one factor maps between shifts of finite type up to almost topological conjugacy.

\section{0 . Introduction}

In [2], shifts of finite type (SFT) were classified up to almost topological conjugacy. The purpose of this paper is to classify finite-to-one continuous factor maps between shifts of finite type. We consider two equivalence relations. The first is very strong: two maps are equivalent if they have the same range shift and they differ by a special kind of almost continuous change of variables in the domain; for a fixed range shift and a fixed generic cardinality of the fibre of the factor maps (a natural invariant), we get infinitely many equivalence classes. The second (more natural) equivalence relation allows the same kind of change of variables in the range (as well as in the domain) and gives only finitely many equivalence classes (for a fixed entropy class and generic cardinality of the fibre). For the latter classification, we reduce the problem to a group action problem which was solved by us in [1]. Our results are completely analogous to and were inspired by those of $D$. Rudolph [10] in the measure-theoretic category (i.e. classification of finite-to-one factor maps between Bernoulli shifts). Our work is basically a more concrete version of Rudolph's work. In particular, let $T$ be a Bernoulli shift whose measure-theoretic entropy $(\log (\lambda))$ is the same as the topological entropy of an aperiodic SFT; then for any finite-to-one factor map of $T$, we construct (theorem 4.3) an equivalent (in the measure-theoretic sense) factor map $\pi: \Sigma_{A} \rightarrow \Sigma_{B}$, where $\Sigma_{B}$ is an arbitrary aperiodic SFT of entropy $\log (\lambda)$ and $\Sigma_{A}$ is some aperiodic SFT of entropy $\log (\lambda)$. (In general, $\Sigma_{A}$ cannot be chosen arbitrarily.)

It would perhaps have been most natural for us to classify our factor maps with respect to continuous changes of variables (i.e. topological conjugacy) since, after all, our factor maps are continuous themselves. But the simplest case of this, when the factor maps involved are identity maps, reduces to the classical topological conjugacy problem for shifts of finite type-which is not yet solved satisfactorily.

For background, we basically refer to [2]. We use the notation $\Sigma_{A}$ for an SFT defined by 0-1 transition matrix $A, \sigma_{A}$ for the shift, and $L_{A}$ for the alphabet (or 
states or symbols) used. The notation $i \rightarrow j$ indicates an allowable transition from symbol $i$ to symbol $j$. We assume that the reader is familiar with the definitions of irreducible (ergodic) and aperiodic (mixing) and also the graph-theoretic description of SFT's.

We shall be interested in continuous factor maps (i.e. onto, continuous, shiftcommuting maps) $\pi: \Sigma_{A} \rightarrow \Sigma_{B}$ between aperiodic SFT's. It is well known that every such map can be represented as a sliding block map which depends on finitely many coordinates. We shall frequently use the fact that every continuous factor map can be recoded to a one-block map (i.e. a sliding block map depending on only one coordinate:

$$
\pi\left(\cdots x_{-1} x_{0} x_{1} \cdots\right)=\cdots \pi^{*}\left(x_{-1}\right) \pi^{*}\left(x_{0}\right) \pi^{*}\left(x_{1}\right) \cdots,
$$

where $\pi^{*}$ is some map $\left.\pi^{*}: L_{A} \rightarrow L_{B}\right)$.

The following is a basic fact:

Proposition 0.1 ([3, theorems 3.3 and 5.7]). Let $\pi: \Sigma_{A} \rightarrow \Sigma_{B}$ be a continuous shiftcommuting map between irreducible SFT's. Then, any two of the following conditions imply the third condition:

(1) $\pi$ is onto ;

(2) $h\left(\Sigma_{A}\right)=h\left(\Sigma_{B}\right)$ (where $h$ denotes topological entropy);

(3) $\pi$ is finite-to-one (i.e. every point has finitely many inverse images).

From the point of view of ergodic theory, every finite-to-one continuous factor map is $k-1$ a.e. for some $k$. (Here 'a.e.' means a.e. with respect to any fully supported ergodic measure, e.g. the unique measure of maximal entropy.) But more is true, as the following indicates:

Proposition 0.2. Let $\pi: \Sigma_{A} \rightarrow \Sigma_{B}$ be a finite-to-one continuous factor map between irreducible SFT's. Then there is a positive integer $k$ such that:

(a) Every point in $\Sigma_{B}$ has at least $k$ mutually separated inverse images (if $\pi$ is a one-block map, then a pair of points $x, \overline{y \in \Sigma_{A}}$ is mutually separated if for all $i, x_{i} \neq y_{i}$ ). (b) Every doubly transitive point in $\Sigma_{B}$ (i.e. a point whose forward and backward orbits are dense) has exactly $k$ inverse images, whence, by (a), all its inverse images are mutually separated.

For the proof of the above, we refer the reader to [3, theorem 6.5]; see also [5]. We also have:

Proposition 0.3. A continuous factor map between irreducible SFT's is finite-to-one if and only if it is $k-1$ a.e. for some $k$.

Proof. (only if) Proposition 0.2 (b) is a strong version of this.

(if) follows from application of $k-1$ a.e. to the measure of maximal entropy to obtain condition (2) of proposition 0.1 .

The following characterizes maps which are constant-to-one.

Proposition 0.4 ([8, Theorem 6.3], see also [5]). A continuous factor map is $k$-to-1 everywhere for some $k$ if and only if it is right and left closing. (Right (left) closing means that the map does not identify two negatively (positively) asymptotic points.) 
For some illuminating examples, we refer the reader to those constructed by $\mathrm{P}$. Shields [10, p. 258].

\section{The strong relation}

In this section, $\Sigma_{A_{1}}, \Sigma_{A_{2}}$ and $\Sigma_{B}$ are aperiodic SFT's all with the same topological entropy. Let $\pi_{1}: \Sigma_{A_{1}} \rightarrow \Sigma_{B}$ and $\pi_{2}: \Sigma_{A_{2}} \rightarrow \Sigma_{B}$ be continuous factor maps.

Definition. We say that $\pi_{1}$ and $\pi_{2}$ are almost topologically conjugate over $\Sigma_{B}$ (A.T.C. over $\Sigma_{B}$ ) if there exists an aperiodic SFT $\Sigma_{D}$ and 1-1 a.e. continuous factor maps

$$
\theta_{i}: \Sigma_{D} \rightarrow \Sigma_{A_{i}} \quad i=1,2
$$

such that the diagram:

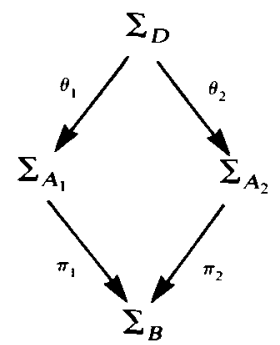

commutes.

Remark. It follows that $\Sigma_{D}$ has the same entropy as $\Sigma_{A_{1}}$ and $\Sigma_{A_{2}}$ and so the maps $\theta_{1}$ and $\theta_{2}$ must be finite-to-one (by proposition 0.1 ).

Definition. The fibred product over $\pi_{1}$ and $\pi_{2}$ is the SFT

$$
\Sigma_{E}=\left\{(x, y) \in \Sigma_{A_{1}} \times \Sigma_{A_{2}}: \pi_{1}(x)=\pi_{2}(y)\right\}
$$

together with the factor maps

$$
\begin{array}{ll}
\psi_{1}: \Sigma_{E} \rightarrow \Sigma_{A_{1}}, & \psi_{1}(x, y)=x ; \\
\psi_{2}: \Sigma_{E} \rightarrow \Sigma_{A_{2}}, & \psi_{2}(x, y)=y .
\end{array}
$$

Note that the diagram

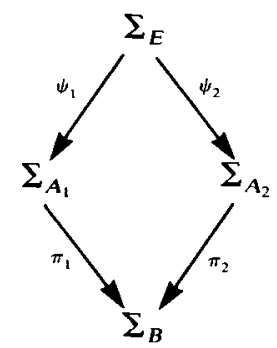

commutes. Also, each $\psi_{i}$ must be finite-to-one.

THEOREM 1.1. The factor maps $\pi_{1}$ and $\pi_{2}$ are A.T.C. over $\Sigma_{B}$ if and only if there is an irreducible component $\Sigma_{E^{\prime}}$ of the fibred product, $\Sigma_{E}$, with maximal entropy (in $\Sigma_{E}$ ) such that the restrictions $\psi_{1} / \Sigma_{E^{\prime}}$ and $\psi_{2} / \Sigma_{E^{\prime}}$ are 1-1 a.e.

Proof. (if) Set $\theta_{i}=\psi_{i}, i=1,2$ and observe that the $\theta_{i}$ must be onto since $\Sigma_{E^{\prime}}$ has full entropy and $\psi_{i}$ are finite-to-one (proposition 0.1 ). Also, $\Sigma_{E^{\prime}}$ is aperiodic since $\psi_{1}$ is 1-1 a.e. and $\Sigma_{A_{1}}$ is aperiodic. 
(only if) Let $\theta_{1}$ and $\theta_{2}$ be the maps that provide the A.T.C. over $\Sigma_{B}$. Now, define the map:

$$
\varphi: \Sigma_{D} \rightarrow \Sigma_{E}, \quad \varphi(z)=\left(\theta_{1}(z), \theta_{2}(z)\right) .
$$

This is well-defined since the diagram (1) commutes. Since $\Sigma_{D}$ is aperiodic, so is its image $\varphi\left(\Sigma_{D}\right)$.

Since the $\theta_{i}$ are assumed to be 1-1 a.e., they are finite-to-one by proposition 0.3 . Thus, $\varphi$ is finite-to-one. So, $\varphi\left(\Sigma_{D}\right)$ has the same entropy as $\Sigma_{D}$, which is the same entropy as $\Sigma_{A_{i}}$ and $\Sigma_{E}$ (since the $\psi_{i}$ and $\theta_{i}$ are finite-to-one and onto). So, $\Sigma_{E} \equiv \varphi\left(\Sigma_{D}\right)$ has maximal entropy in $\Sigma_{E}$; since $\Sigma_{E^{\prime}}$ is also aperiodic, it follows that $\Sigma_{E^{\prime}}$ is an irreducible component of maximal entropy in $\Sigma_{E}$.

Moreover the diagram:

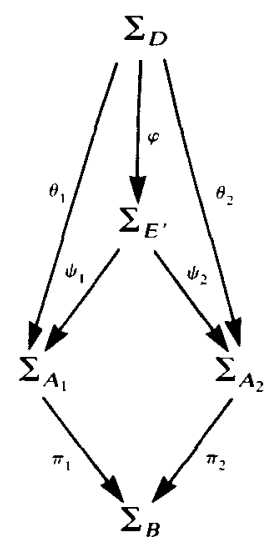

commutes. Now, since $\theta_{i}$ are 1-1 a.e. $i=1,2$, it follows that $\psi_{i} \mid \Sigma_{E^{\prime}}$ are 1-1 a.e. $i=1,2$ as desired.

Remarks. (i) The classification given above is completely effective since: (1) the construction of the fibred product is constructive (assuming by recoding that $\pi_{1}$ and $\pi_{2}$ are 1-block maps, then $\Sigma_{E}$ is the SFT with alphabet

$$
L_{E}=\left\{\left(a_{1}, a_{2}\right) \in L_{A_{i}} \times L_{A_{2}}: \pi_{1}\left(a_{1}\right)=\pi_{2}\left(a_{2}\right)\right\} \text {, }
$$

and transitions $\left(a_{1}, a_{2}\right) \rightarrow\left(a_{1}^{\prime}, a_{2}^{\prime}\right)$ if and only if $a_{1} \rightarrow a_{1}^{\prime}$ and $\left.a_{2} \rightarrow a_{2}^{\prime}\right)$; (2) the determination of irreducible components is constructive; and (3) there is a finite procedure for deciding whether or not a factor map is $1-1$ a.e. (see $[4,3.4]$ ).

(ii) The generic cardinality of the fibre for a finite-to-one continuous factor map (which always exists (proposition 0.2)), is clearly an invariant of A.T.C. over $\Sigma_{B}$ (i.e. if $\pi_{1}$ and $\pi_{2}$ are A.T.C. over $\Sigma_{B}$ and $\pi_{1}$ is $k_{1}$-to-1 a.e. and $\pi_{2}$ is $k_{2}$-to-1 a.e., then $k_{1}=k_{2}$ ).

(iii) From a measure-theoretic point of view, the factor map $\pi_{1}: \Sigma_{A_{1}} \rightarrow \Sigma_{B}$ decomposes $\Sigma_{A_{1}}$ as a skew-product over $\Sigma_{B}$ with $k$-point fibres (where $k$ is the generic cardinality of the fibre): namely, $\Sigma_{A_{1}}$ can be written as $\Sigma_{B} \times\{1, \ldots, k\}$ and the shift $\sigma_{A_{1}}$ can be represented as

$$
\sigma_{A_{1}}(x, i)=\left(\sigma_{B}(x), f_{1}(x)(i)\right)
$$


where $f_{1}: \Sigma_{B} \rightarrow S_{k}$ is a measurable (in fact, almost continuous) map into the symmetric group on $k$ letters. Here, the measure structure is given by the measure of maximal entropy, and almost continuous means continuous on some subset of full measure. Similarly, we get a function $f_{2}: \Sigma_{B} \rightarrow S_{k}$ for the map $\pi_{2}$.

Now, one can easily show that if $\pi_{1}$ and $\pi_{2}$ are A.T.C. over $\Sigma_{B}$, then there exists a measurable (in fact, almost continuous) map $h: \Sigma_{B} \rightarrow S_{k}$ such that the cohomology equation:

$$
f_{1}=\left(h \circ \sigma_{B}\right) \cdot f_{2} \cdot h^{-1}
$$

holds a.e., (where for $x \in \Sigma_{B}, h^{-1}(x)$ denotes the inverse permutation of $h(x)$ ) (see $[10$, p. 256]).

(iv) If $\pi_{1}$ and $\pi_{2}$ are $k$-to-1 everywhere, then $f_{1}$ and $f_{2}$ can (in (2)) be chosen continuous and in fact depend on only finitely many coordinates (see [8]). It follows then that the solution $h$ to (3) (if it exists) must depend on only finitely many coordinates as well, and in fact (3) holds everywhere. The proof of this is similar to the results [6, theorem 1 and remark 2] [9, 2.42] and is deferred to the appendix. The continuity of $h$ then yields:

Proposition 1.2. If two k-to-1 everywhere extensions $\pi_{1}$ and $\pi_{2}$ of $\Sigma_{B}$ are A.T.C. over $\Sigma_{B}$, then in fact they are topologically conjugate over $\Sigma_{B}$ (i.e. there is a topological conjugacy $\bar{h}: \Sigma_{A_{1}} \rightarrow \Sigma_{A_{2}}$ such that $\pi_{1}=\pi_{2} \circ \bar{h}$ ).

From this, one can use the form (2) to construct, for each SFT $\Sigma_{B}$ and integer $k \geq 2$, infinitely many inequivalent (in the sense of A.T.C. over $\Sigma_{B}$ ) $k$-to-1 everywhere extensions of $\Sigma_{B}$. (Just play with the zeta functions of the domains.)

2. The weak relation: reduction to constant-to-one maps

In this and the next section, $\Sigma_{A_{1}}, \Sigma_{A_{2}}, \Sigma_{B_{1}}$ and $\Sigma_{B_{2}}$ are aperiodic SFT's of the same entropy. Let $\pi_{1}: \Sigma_{A_{1}} \rightarrow \Sigma_{B_{1}}$ and $\pi_{2}: \Sigma_{A_{2}} \rightarrow \Sigma_{B_{2}}$ be continuous onto factor maps (hence finite-to-one).

Definition. We say that $\pi_{2}$ is an almost topological factor of $\pi_{1}$ (and $\pi_{1}$ is an almost topological extension of $\pi_{2}$ ) if there exist 1-1 a.e. continuous factor maps $\theta: \Sigma_{A_{1}} \rightarrow \Sigma_{A_{2}}$ and $\rho: \Sigma_{B_{1}} \rightarrow \Sigma_{B_{2}}$ such that the diagram:

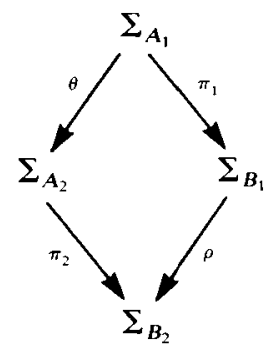

commutes.

Definition. We say that $\pi_{1}$ and $\pi_{2}$ are almost topologically conjugate (A.T.C.) if they have a common almost conjugate extension (cf. [2]). 
We show, in this section, that we may as well assume that our factor maps are constant-to-one (i.e. $k$-to-1 everywhere for some $k$ ).

THEOREM 2.1. Let $\Sigma_{A}$ and $\Sigma_{B}$ be aperiodic SFT's and let $\pi: \Sigma_{A} \rightarrow \Sigma_{B}$ be a k-to-1 a.e. continuous factor map. Then there is an almost topological extension of $\pi$ which is k-to-1 everywhere.

Proof. We may assume that $\pi$ is a 1-block map by the usual recoding argument.

Define $\Sigma_{B^{\prime}}$ as follows: the alphabet of $\Sigma_{B^{\prime}}$ is the set of all distinct unordered $k$-tuples of symbols from $L_{A}$ with the same $\pi$-image:

$L_{B^{\prime}}=\left\{\left\{a_{1}, \ldots, a_{k}\right\}: a_{1}, \ldots, a_{k} \in L_{A}\right.$ are distinct and $\left.\pi\left(a_{1}\right)=\pi\left(a_{2}\right)=\cdots=\pi\left(a_{k}\right)\right\}$ and transitions

$$
\left\{a_{1}, \ldots, a_{k}\right\} \rightarrow\left\{a_{1}^{\prime}, \ldots, a_{k}^{\prime}\right\}
$$

if and only if there is a permutation $q \in S_{k}$ such that for each $i=1, \ldots, k$

$$
a_{i} \rightarrow a_{q(i)}^{\prime} \quad \text { in } \Sigma_{A} \text {. }
$$

Let $\rho: \Sigma_{B^{\prime}} \rightarrow \Sigma_{B}$ be defined in the obvious manner; namely:

$$
\rho\left(\left\{a_{1}, \ldots, a_{k}\right\}\right)=\pi\left(a_{1}\right) .
$$

Then Coven and Paul $([4,3.4])$ proved that there is an irreducible component of $\Sigma_{B^{\prime}}$ such that the restriction of $\rho$ to this component is finite-to-one, onto and 1-1 a.e. (We will abuse notation and call this component $\Sigma_{B^{\prime}}$ as well.)

Now let $\Sigma_{A^{\prime}}$ be the SFT defined by

$$
L_{A^{\prime}}=\left\{\left(a,\left\{a_{1}, \ldots, a_{k}\right\}\right): a \in L_{A},\left\{a_{1}, \ldots, a_{k}\right\} \in L_{B^{\prime}} \text { and } a \in\left\{a_{1}, \ldots, a_{k}\right\}\right\}
$$

with transitions:

if and only if

$$
\left(a,\left\{a_{1}, \ldots, a_{k}\right\}\right) \rightarrow\left(a^{\prime},\left\{a_{1}^{\prime}, \ldots, a_{k}^{\prime}\right\}\right)
$$

$$
a \rightarrow a^{\prime} \text { and }\left\{a_{1}, \ldots, a_{k}\right\} \rightarrow\left\{a_{1}^{\prime}, \ldots, a_{k}^{\prime}\right\} .
$$

Define $\psi_{1}: \Sigma_{A^{\prime}} \rightarrow \Sigma_{A}$ by $\psi_{1}(x, y)=x$ and $\psi_{2}: \Sigma_{A^{\prime}} \rightarrow \Sigma_{B^{\prime}}$ by $\psi_{2}(x, y)=y$. (Note that we have here a subshift of the fibred product over $\pi$ and $\rho$.)

Of course the diagram:

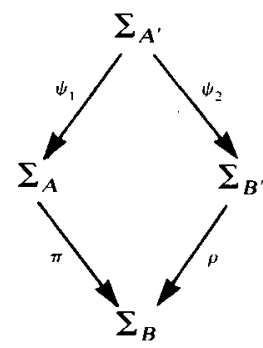

commutes.

Since $\pi$ and $\rho$ are finite-to-one and onto, so are $\psi_{1}$ and $\psi_{2}$. So, if we replace $\Sigma_{\boldsymbol{A}^{\prime}}$ by any one of its irreducible components of maximal entropy (which we will still call $\Sigma_{A^{\prime}}$ ) we can assume (by proposition 0.1 ) that $\Sigma_{A^{\prime}}$ is irreducible and that $\psi_{1}$ and $\psi_{2}$ are finite-to-one and onto. Moreover, since $\rho$ is $1-1$ a.e. so is $\psi_{1}$. So, the factor map $\psi_{2}$ is an almost topological extension of $\pi$. It remains to show that $\psi_{2}$ is $k$-to- 1 
everywhere. The proof of this follows from the following:

LEMMA 2.2. If $\left\{a_{1}, \ldots, a_{k}\right\} \rightarrow\left\{a_{1}^{\prime}, \ldots, a_{k}^{\prime}\right\}$ in $\Sigma_{B^{\prime}}$ then for all $i=1, \ldots, k$ there exists a unique $j \in\{1, \ldots, k\}$ such that $a_{i} \rightarrow a_{j}^{\prime}$.

Proof. By definition of the transition

$$
\left\{a_{1}, \ldots, a_{k}\right\} \rightarrow\left\{a_{1}^{\prime}, \ldots, a_{k}^{\prime}\right\}
$$

for each $i$, there exists $j$ such that

$$
a_{i} \rightarrow a_{j}^{\prime}
$$

Suppose there were two such $j$ (call them $j_{1}$ and $j_{2}$ ). Let $w$ be a doubly transitive point in $\Sigma_{B^{\prime}}$. Assume also that

$$
w_{0}=\left\{a_{1}, \ldots, a_{k}\right\}, \quad w_{1}=\left\{a_{1}^{\prime}, \ldots, a_{k}^{\prime}\right\} .
$$

Then $\rho(w)$ is a doubly transitive point in $\Sigma_{B}$ and $\pi^{-1}(\rho(w))$ contains two mutually separated points $x$ and $y$ where

But then the point $u$ defined by

$$
x_{0}=a_{i}, \quad x_{1}=a_{j_{1}}^{\prime}, \quad y_{1}=a_{j_{2}}^{\prime} .
$$

$$
u_{l}= \begin{cases}x_{l} & \text { if } l \leq 0, \\ y_{l} & \text { if } l>0,\end{cases}
$$

is also in $\pi^{-1}(\rho(w))$; also $u$ is not mutually separated from $x$ (nor $y$ ); this contradicts the fact (proposition 0.2 ) that the inverse images of a doubly transitive point (in this case $\rho(w))$ are mutually separated. This completes the proof of lemma 2.2 .

One might ask for more than the preceding result yields: namely, if $\pi: \Sigma_{A} \rightarrow \Sigma_{B}$ is a $k$-to- 1 a.e. factor map, then can $\pi$ be written as

$$
\pi=\pi_{1} \circ \pi_{2}
$$

where the (range of $\pi_{2}$ ) $=$ (domain of $\pi_{1}$ ) is an SFT and

(i) $\pi_{1}$ is $k$-to- 1 everywhere? or

(ii) $\pi_{2}$ is $k$-to- 1 everywhere?

The following examples show neither of these to be true.

Example 1. Let $\Sigma_{A}$ be the SFT defined by the graph:

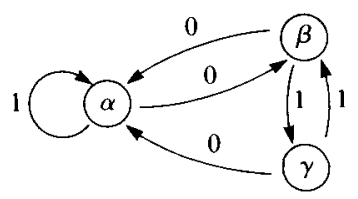

The labels on the edges define a factor map $\pi: \Sigma_{A} \rightarrow \Sigma_{B}$ where $\Sigma_{B}$ is the full 2-shift. The map is $2-1$ a.e. since the collapsing of vertices $\beta$ and $\gamma$ decomposes $\pi$ into a 1-1 a.e. map followed by a 2-to-1 map.

However, if $\pi$ could be factored $\pi=\pi_{2}{ }^{\circ} \pi_{1}$ where $\pi_{1}$ was 2 -to-1 everywhere, then every fibre of $\pi$ would have an even number of points. But $\pi^{-1}(\ldots 1111 \ldots)$ has 3 points. 
Example 2. Let $\Sigma_{A}$ and $\Sigma_{B}$ be the SFT's defined by:

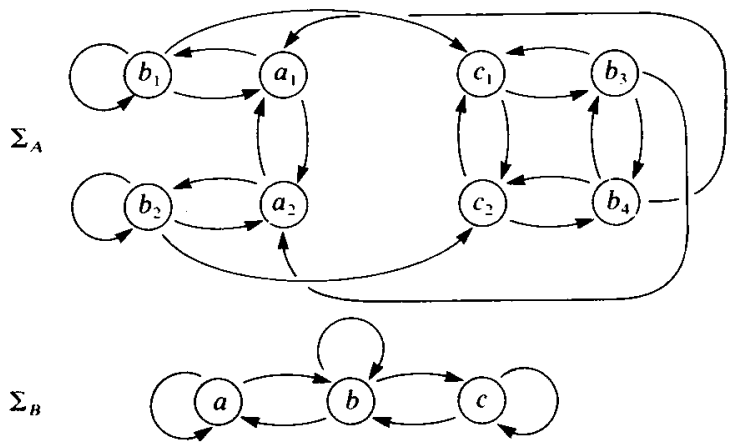

The map $\pi: \Sigma_{A} \rightarrow \Sigma_{B}$ is defined by 'dropping subscripts'. One easily sees that this map is 2-to-1 a.e. Suppose that $\pi$ factored as $\pi=\pi_{2}{ }^{\circ} \pi_{1}$ where $\pi_{2}$ was 2-to-1 everywhere. Then, by proposition $0.4, \pi_{2}$ would be left closing. Then, $\pi_{1}$ must identify the following 2 pairs of points:

$$
\begin{aligned}
& \ldots b_{1} b_{1} b_{1} b_{1} c_{1} c_{2} c_{1} c_{2} \ldots \text { and } \ldots b_{4} b_{3} b_{4} b_{3} c_{1} c_{2} c_{1} c_{2} \ldots \\
& \ldots b_{2} b_{2} b_{2} b_{2} c_{2} c_{1} c_{2} c_{1} \ldots \text { and } \ldots b_{3} b_{4} b_{3} b_{4} c_{2} c_{1} c_{2} c_{1} \ldots
\end{aligned}
$$

But then by the continuity of $\pi_{1}$, it must also identify the pairs of points:

$$
\begin{aligned}
& \ldots b_{1} b_{1} b_{1} b_{1} \ldots \text { and } \ldots b_{4} b_{3} b_{4} b_{3} \ldots \text {; } \\
& \ldots b_{2} b_{2} b_{2} b_{2} \ldots \text { and } \ldots b_{3} b_{4} b_{3} b_{4} \ldots
\end{aligned}
$$

Thus, $\pi_{1}$ must identify all the points in the $\pi$-fibre of $\bar{b}=\ldots b b b \ldots$ So, $\pi_{2}^{-1}(\bar{b})$ is a single point. But this contradicts the assumption that $\pi_{2}$ was 2-to-1 everywhere.

Finally we remark that the direct product map of the factor maps $\pi$ constructed in examples 1 and 2 provides an example of a 4-to-1 a.e. map which cannot be factored: $\pi=\pi_{2} \circ \pi_{1}$ so that either $\pi_{1}$ or $\pi_{2}$ is 4-to-1 everywhere.

\section{The weak relation: reduction to group actions}

In this section, we reduce the classification of factor maps to the classification of certain group actions (theorem 3.1 below); this is very much analogous to [10]. However, our group actions are continuous, a fact that follows basically from theorem 2.1.

Let $\Sigma_{A}$ and $\Sigma_{B}$ be irreducible SFT's of the same entropy and let $\pi: \Sigma_{A} \rightarrow \Sigma_{B}$ be a $k$-to-1 everywhere factor map. As mentioned before ( $\$ 1$, remark (iv)), up to a topological conjugacy of $\pi, \Sigma_{A}$ can be written as a continuous skew-product over $\Sigma_{B}$ and, in fact, the skewing function depends on finitely many coordinates; by recoding, we can actually assume that the skewing function depends on only two coordinates: i.e.

and in this form

$$
\Sigma_{A}=\Sigma_{B} \times\{1, \ldots, k\}
$$

$$
\begin{aligned}
\pi(x, i) & =x, \\
\sigma_{A}(x, i) & =\left(\sigma_{B}(x), f\left(x_{0} x_{1}\right)(i)\right),
\end{aligned}
$$

where $f$ is some function on 2-blocks taking values in $S_{k}$ (the symmetric group). 
So $L_{A}=L_{B} \times\{1, \ldots, k\}$ and the transitions are given by

$$
(b, i) \rightarrow\left(b^{\prime}, j\right)
$$

if and only if

$$
b \rightarrow b^{\prime} \text { in } \Sigma_{B} \text { and } j=f\left(b b^{\prime}\right)(i) .
$$

As in [10], we introduce the full extension of $\pi$ as the SFT

$$
\begin{gathered}
\Sigma_{\pi}=\Sigma_{B} \times S_{k}, \\
\sigma_{\pi}(x, g)=\left(\sigma_{B}(x), f\left(x_{0} x_{1}\right) g\right) .
\end{gathered}
$$

Definition. Let $G$ be a finite group and $\Sigma$ an SFT. A $\mathbb{Z} \times G$ action on $\Sigma$ is the action of the shift together with a continuous action of the group $G$ on $\Sigma$ in which each element of $G$ commutes with the shift. We assume that the $G$ part of the action is free, i.e. that no non-trivial element of $G$ fixes any element of $\Sigma$.

The $\mathbb{Z} \times G$ action we are most interested in is the following: let $S_{k}$, the symmetric group on $k$ letters, act on $\Sigma_{\pi}$ by its natural action on the alphabet of $\Sigma_{\pi}$ namely, $\bar{g}(x, g)=(x, g \bar{g})$. We call this the full $\mathbb{Z} \times S_{k}$ action on $\Sigma_{r}$. We let $G$ act on the right since the shift $\sigma_{\pi}$ acts on the left. (This makes the $G$-action commute with $\sigma_{\pi}$ )

Definition. Let $G$ be a finite group and let $\Sigma_{1}$ and $\Sigma_{2}$ be two SFT's, each with a $\mathbb{Z} \times G$ action. We say that these two $\mathbb{Z} \times G$ actions are almost topologically conjugate (A.T.C.) if there is another SFT $\Sigma_{3}$ with a $\mathbb{Z} \times G$ action and $1-1$ a.e. continuous factor maps $\Sigma_{3} \rightarrow \Sigma_{1}$ and $\Sigma_{3} \rightarrow \Sigma_{2}$ which also commute with the $G$-actions.

The reason for our interest in group actions is the following:

THEOREM 3.1. Let $\pi_{1}: \Sigma_{A_{1}} \rightarrow \Sigma_{B_{1}}$ and $\pi_{2}: \Sigma_{A_{2}} \rightarrow \Sigma_{B_{2}}$ be two $k$-to-1 everywhere continuous

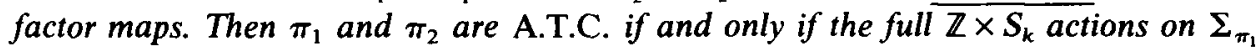
and $\Sigma_{\pi_{2}}$ are A.T.C.

Proof. (only if) Let $\pi_{1}$ and $\pi_{2}$ be A.T.C. and let $\pi_{3}: \Sigma_{A_{3}} \rightarrow \Sigma_{B_{3}}$ be the common almost topological extension of $\pi_{1}$ and $\pi_{2}$. Clearly, $\pi_{3}$ is $k$-to-1 a.e. We may assume by theorem 2.1 that $\pi_{3}$ is in fact $k$-to- 1 everywhere and that all maps $\pi_{1}, \pi_{2}, \pi_{3}$ are in the form (4) with $\Sigma_{A_{i}}$ built with skewing function $f_{i}$ over $\Sigma_{B_{i}}$.

We have the commutative diagram:

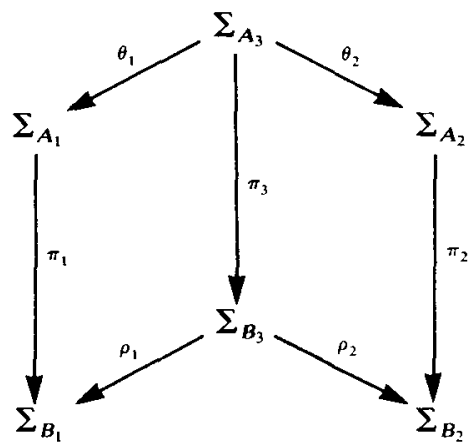

Since $\pi_{1}, \pi_{2}$ and $\pi_{3}$ are simply projections onto the first coordinate of (4), we have

$$
\theta_{1}(x, i)=\left(\rho_{1} x, l_{1}(x)(i)\right)
$$


and

$$
\theta_{2}(x, i)=\left(\rho_{2} x, l_{2}(x)(i)\right)
$$

for some $l_{1}, l_{2}: \Sigma_{B_{3}} \rightarrow S_{k}$. Clearly, $l_{1}$ and $l_{2}$ are continuous (since $\rho_{i}, \theta_{i}$ are). Now define

$$
\bar{\theta}_{1}: \Sigma_{\pi_{3}} \rightarrow \Sigma_{\pi_{1}}, \quad \bar{\theta}_{1}(x, g)=\left(\rho_{1} x, l_{1}(x) g\right),
$$

and

$$
\bar{\theta}_{2}: \Sigma_{\pi_{3}} \rightarrow \Sigma_{\pi_{1}}, \quad \bar{\theta}_{2}(x, g)=\left(\rho_{2} x, l_{2}(x) g\right) .
$$

We leave it to the reader to verify that

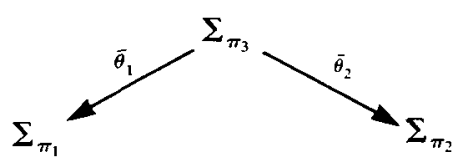

is an A.T.C. of group actions.

For the converse, let $\Sigma_{3}$ be a common almost topological extension of $\Sigma_{\pi_{1}}$ and $\Sigma_{\pi_{2}}$ (in the $\mathbb{Z} \times S_{k}$ category). We would like to produce a continuous factor map $\pi_{3}$ for which the full action is $\Sigma_{3}$. This is done as follows: we construct two equivalence relations on $\Sigma_{3}$ :

$$
\begin{aligned}
& x \sim y \quad \text { if there exists } g \in S_{k} \text { such that } g x=y, \\
& x \approx y \quad \text { if there exists } g \in S_{k} \text { such that } g x=y \text { and } g(1)=1 .
\end{aligned}
$$

(When we say $g(1)=1$, we are thinking of $g$ acting on $\{1, \ldots, n\}$.) Let

$$
\begin{aligned}
& \Sigma_{A_{3}}=\Sigma_{3} / \approx \\
& \Sigma_{B_{3}}=\Sigma_{3} / \sim
\end{aligned}
$$

and let $\pi_{3}: \Sigma_{A_{3}} \rightarrow \Sigma_{B_{3}}$ be the obvious map. One can verify that $\Sigma_{A_{3}}$ and $\Sigma_{B_{3}}$ are indeed SFT's. Moreover, since the continuous factor maps $\Sigma_{3} \rightarrow \Sigma_{\pi_{1}}$ and $\Sigma_{3} \rightarrow \Sigma_{\pi_{2}}$ commute with the $S_{k}$ actions, they naturally induce 1-1 a.e. maps $\theta_{1}, \rho_{1}, \theta_{2}, \rho_{2}$ such that the diagram (5) above commutes, i.e. an A.T.S. between $\pi_{1}$ and $\pi_{2}$.

Finally, we mention that the work in this section can be viewed in a slightly more concrete manner:

Namely, $\Sigma_{\pi}$ can be defined as the SFT whose alphabet is the set of ordered $k$-tuples $\left(b_{1}, \ldots, b_{k}\right)$ where $b_{1}, \ldots, b_{k}$ are distinct elements of $L_{A}$ all with the same $\pi$-image, $b$ in $L_{B}$ (the transition are the ones naturally inherited from $\Sigma_{A}$ ). In this setting, $S_{k}$ acts by permuting the ordering.

\section{Construction of factor maps}

Theorems 2.1 and 3.1, together with theorem 4.2 below, will give an effective procedure for classifying finite-to-one factor maps between shifts of finite type up to A.T.C. In particular, for a fixed entropy class and a fixed generic cardinality of the fibre, we will show that there are only finitely many equivalence classes all defined by the algebraic invariants of $[\mathbf{1 0}]$.

We first recall the results of [1]. Consider an irreducible SFT $\Sigma_{C}$, with period $p=p_{C}$. This means that $\Sigma_{C}$ can be written as a disjoint union of $p$ subsets $\Sigma_{C}^{i}$, 
$i=0, \ldots, p-1$ such that

(i) $\sigma\left(\Sigma_{C}^{i}\right)=\Sigma_{C}^{(i+1)(\bmod p)}$; and

(ii) $\sigma^{p} / \Sigma_{C}^{0}$ is aperiodic.

For a $\mathbb{Z} \times G$ action on $\Sigma_{C}$ one defines

$$
H_{C}^{i}=\left\{g \in G: g\left(\Sigma_{C}^{0}\right)=\Sigma_{C}^{i}, i=0, \ldots, p-1\right\} .
$$

Note that $g\left(\Sigma_{C}^{j}\right)=\Sigma_{C}^{(i+j) \bmod p}$ for all $g \in H_{C}^{i}$ and $j \in\{0, \ldots, p-1\}$. The main result of [1] is:

THEOREM 4.1. ([1, theorem 6]) Let $\Sigma_{C_{1}}$ and $\Sigma_{C_{2}}$ be irreducible SFT's. Two $\mathbb{Z} \times G$ actions, one on $\Sigma_{C_{1}}$ and the other on $\Sigma_{C_{2}}$, are A.T.C. if and only if

(a) $h\left(\Sigma_{C_{1}}\right)=h\left(\Sigma_{C_{2}}\right)$

(b) $p_{C_{1}}=p_{C_{2}}$; and

(c) for all $i, H_{C_{1}}^{i}=H_{C_{2}}^{i}$.

We now restate this result in a slightly different form (similar to [10]). First observe that, since the $G$-part of a $\mathbb{Z} \times G$ action on $\Sigma_{C}$ commutes with the shift, $H_{C}^{0}$ is a normal subgroup of $G$. Next observe that if $g_{C} \in H_{C}^{1}$, then for each $i=0, \ldots, p-1$ (where $p=p_{C_{1}}=p_{C_{2}}$ )

$$
H_{C}^{i}=\left(g_{C}\right)^{i} H_{C}^{0}
$$

So, each $H_{C}^{i}$ is actually a coset of $H_{C}^{0}$; since $g_{C}^{p} \in H_{C}^{0}$ it follows that $G / H_{C}^{0}$ is a cyclic group with generator $g_{C} H_{C}^{0}$.

Now, suppose for two $\mathbb{Z} \times G$ actions:

(c') $H_{C_{1}}^{0}=H_{C_{2}}^{0}$ and $g_{C_{1}}=g_{C_{2}} \bmod \left(H_{C_{1}}^{0}\right)$.

This is equivalent to saying

$$
H_{C_{1}}^{0}=H_{C_{2}}^{0} \text { and } H_{C_{1}}^{1}=H_{C_{2}}^{1} .
$$

Then we also get

$$
H_{C_{1}}^{i}=\left(g_{C_{1}}\right)^{i} H_{C_{1}}^{0}=\left(g_{C_{2}}\right)^{i} H_{C_{2}}^{0}=H_{C_{2}}^{i}
$$

which is condition (c). So, condition ( $\left.c^{\prime}\right)$ can replace condition (c) in theorem 4.1.

Unfortunately, the SFT's we must consider may be reducible. However, they do satisfy a weaker property:

Definition. An SFT $\Sigma_{C}$ is non-wandering if it is a disjoint union of irreducible components.

Note. In general, an SFT may have points that 'wander' from one component to another (see $[2$, p. 21]).

For any $\mathbb{Z} \times G$ action on a non-wandering SFT, the $G$-part of the action acts on the set of irreducible components of the SFT. If this $G$ action is transitive, we say that the $\mathbb{Z} \times G$ action is $G$-transitive.

For a non-wandering SFT, $\Sigma_{C}$, and a $G$-transitive $\mathbb{Z} \times G$ action on $\Sigma_{C}$, pick an arbitrary irreducible component $\Sigma_{\bar{C}}$ of $\Sigma_{C}$ and let $G_{\bar{C}}=\left\{g \in G: g\left(\Sigma_{\bar{C}}\right)=\Sigma_{\bar{C}}\right\}$. Define 
$H_{C}^{0}$ and $g_{C}$ as $H_{\bar{C}}^{0}$ and $g_{\bar{C}}$ (resp.) where the latter are defined as above for the restricted $\mathbb{Z} \times G_{\bar{C}}$ action on $\Sigma_{\bar{C}}$. Now, $H_{C}^{0}=H_{\bar{C}}^{0}$ is normal in $G_{\bar{C}}$, but not necessarily normal in $G$. However, $g_{C} \in N\left(H_{C}^{0}\right\}$, the $G$-normalizer of $H_{C}^{0}$.

Now, suppose one picks two different components $\Sigma_{\bar{C}}$ and $\Sigma_{\hat{C}}$ of $\Sigma_{C}$. Let $g \in G$ map $\Sigma_{\bar{C}}$ onto $\Sigma_{\hat{C}}$; then

$$
g H_{\bar{C}} g^{-1}=H_{\hat{C}}
$$

and

$$
g\left(g_{\bar{C}} H_{\bar{C}}\right) g^{-1}=g_{\hat{C}} H_{\hat{C}} .
$$

The latter is equivalent to:

$$
g g_{\bar{C}} g^{-1}=g_{\hat{C}} \bmod H_{\hat{C}} .
$$

So, our choices of $H_{C}^{0}$ and $g_{C}$ are unique only up to conjugation in $G$ (and $g_{C}$ is unique only up to that conjugation plus multiplication by an element of $H_{C}^{0}$ ). Using these observations, the following is straightforward:

THEOREM 4.2. Let $\Sigma_{C_{1}}$ and $\Sigma_{C_{2}}$ be two non-wandering SFT's each with a transitive $\mathbb{Z} \times G$ action. Then these actions are A.T.C. if and only if:

(A) $h\left(\Sigma_{C_{1}}\right)=h\left(\Sigma_{C_{2}}\right)$;

(B) $H_{C_{1}}^{0}$ is conjugate to $H_{C_{2}}^{0}$ (by a conjugacy $g$ );

(C) $\mathrm{gg}_{C_{1}} g^{-1}=g_{C_{2}} \bmod H_{C_{2}}^{0}$.

We leave it to the reader (see [10]), to see that the full action for a factor map between aperiodic SFT's of the same entropy satisfies the above hypothesis: i.e. $\Sigma_{\pi}$ is non-wandering and the full action is $G$-transitive. Now we prove:

THEOREM 4.3. For any aperiodic SFT $\Sigma_{B}$ and any subgroup $H$ of $S_{k}(k \in \mathbb{N})$ and any element $g \in N(H)$, there is a factor map $\pi: \Sigma_{A} \rightarrow \Sigma_{B}$ where $\Sigma_{A}$ is an SFT and $\pi$ is $k-1$ everywhere and the full $\mathbb{Z} \times S_{k}$ action on $\Sigma_{\pi}$ realizes the invariants $H$ and $g$ (i.e. there is an irreducible component $\Sigma_{C}$ of $\Sigma_{\pi}$ for which $H_{C}^{0}=H$ and $g_{C}=g$ ).

One can show, as Rudolph did ([10, theorem 2]), that if $H$ acts transitively on $\{1, \ldots, k\}$, then $\Sigma_{A}$ can be chosen aperiodic. The reader should compare theorem 4.3 with the construction given at the end of $[10]$.

Question. Given $k, H$ and $g$, for which SFT's $\Sigma_{A}$ does there exist an SFT $\Sigma_{B}$ and $k$-to-1 a.e. factor map $\pi: \Sigma_{A} \rightarrow \Sigma_{B}$ realizing the invariant $H$ and $g$ ? Arguments in Hedlund ([5]) can be used to show that for $k=3$ and $\Sigma_{A}=$ (the full 2-shift) no such map exists (for any choice of $H$ and $g$ ).

The proof of theorem 4.3 follows the lines of [10]. First we need:

LEMMA 4.4. Let $\Sigma_{B}$ be an aperiodic SFT. Let $n$ be a positive integer. Then there is an SFT, topologically conjugate to $\Sigma_{B}$, which has a state with at least $n$ successors (i.e. outgoing transitions).

Proof. We do this by induction on $n$. For $n=2$, this follows immediately from the fact that $\Sigma_{B}$ is aperiodic. Now, assume that $\Sigma_{B}$ has a state $v$ with $n \geq 2$ successors; 
we will produce a conjugate SFT which has a state with at least $n+1$ successors. Let $\eta=\eta_{1} \eta_{2} \cdots \eta_{t}$ be a simple path (in the defining graph for $\Sigma_{B}$ ) where $\eta_{1}$ has at least two predecessors (i.e. incoming transitions) and $\eta_{t}=v$. The existence of $\eta$ follows from aperiodicity. By a complete splitting of the state $v$, we mean a new graph defined by splitting the vertex $v$ into new vertices, one for each successor of $v$ (see [7] for the notion of state splitting); here, each predecessor (in particular, $\left.\eta_{t-1}\right)$ of $v$ is a predecessor of each of the new vertices, and each new vertex has exactly one successor, namely the successor that it represents.
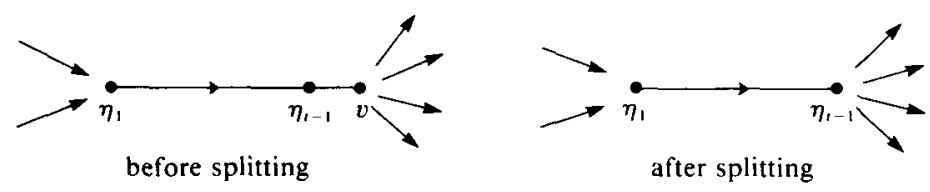

The SFT defined by this new graph is naturally conjugate to $\Sigma_{B}$ and the state $\eta_{t-1}$ now has at least $n$ successors. So, by a conjugacy, we have effectively reduced the length of the path $\eta$. By continuing this inductively downward (shortening $\eta$ ), we obtain an SFT conjugate to $\Sigma_{B}$ which has a state $v$ with at least $n$ successors and 2 predecessors. A complete splitting of $v$ now gives us two distinct vertices $v_{1}, v_{2}$ each with at least $n$ successors. Now, let $\eta^{\prime}$ be a simple path in the defining graph of the new SFT which originates at a successor $s$ of $v_{1}$ and terminates at $v_{2}$.

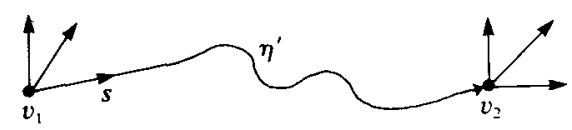

We may assume that $\eta^{\prime}$ does not pass through $v_{1}$ or any successors of $v_{1}$ (other than $s$ ). Again, we can do a complete splitting of $v_{2}$ and thereby effectively reduce the length of $\eta^{\prime}$; continuing inductively downward (shortening $\eta^{\prime}$ ), we can assume that $v_{2}$ is a successor of $v_{1}$. Now, a complete splitting of $v_{2}$ will provide $v_{1}$ with at least $2 n-1 \geq n+1$ successors as desired.

We apply the lemma as follows. Let $n$ be the order of $H$ and write

$$
H=\left\{h_{1}, \ldots, h_{n}\right\}
$$

Let $m$ be the order of $g$ in $S_{k}$ and let $p$ be the order of $g H$ in $N(H) / H$.

By virtue of the lemma, we may as well assume that $\Sigma_{B}$ itself has a state $v$ with at least $n+2$ successors, $s_{1}, \ldots, s_{n+2}$. Moreover, using the aperiodicity assumption and replacing $\Sigma_{B}$ by some higher-block presentation (see [2, p. 13]) of itself, we may assume that there exist cycles $\gamma_{1}, \ldots, \gamma_{n}, \alpha_{1}, \alpha_{2}$ such that:

(1) the initial edges of $\gamma_{1}, \ldots, \gamma_{n}, \alpha_{1}, \alpha_{2}$ are the edges $v s_{1}, \ldots, v s_{n+2}$;

(2) the set of noninitial edges of $\gamma_{1}, \ldots, \gamma_{n}, \alpha_{1}, \alpha_{2}$ is disjoint from the set of initial edges;

(3) the lengths of $\gamma_{1}, \ldots, \gamma_{n}$ are multiples of $m$; and

(4) the lengths of $\alpha_{1}, \alpha_{2}$ are multiples of $p$ by a pair of relatively prime numbers. 


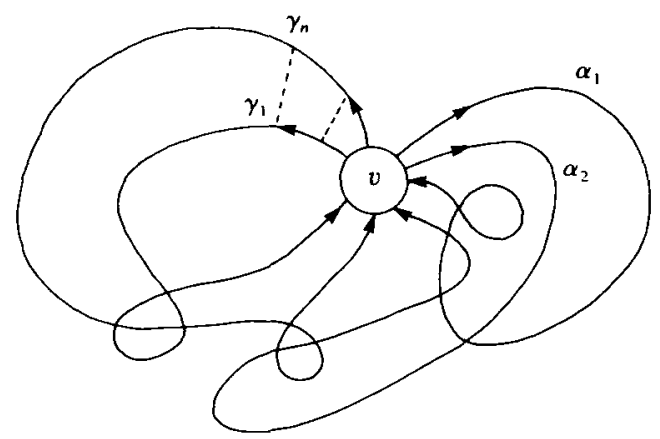

(We leave it to the reader to verify this.) Now, define $f$ on the 2-blocks of $\Sigma_{B}$ by:

$$
f\left(b b^{\prime}\right)=\left\{\begin{array}{lll}
g h_{i} & \text { if } b b^{\prime}=v s_{i}, & 1 \leq i \leq n \\
(g)^{1-\left|\alpha_{i}\right|} & \text { if } b b^{\prime}=v s_{n+i}, & i=1,2 \\
g & \text { otherwise. }
\end{array}\right.
$$

(Here, $\left|\alpha_{i}\right|$ denotes the length of the cycle $\alpha_{i}$ ) Note that since $\left|\alpha_{i}\right|$ is a multiple of $p$, the order of $g H$ in the group $N(H) / H$, it follows that $(g)^{-\left|\alpha_{i}\right|}$ belongs to $H$. So, the range of $f$ lies in the coset $g H$. Using this $f$, let $\pi$ be the map defined as in (4) of $\S 3$.

We will show that $\Sigma_{B} \times H$ is a cyclic subset for an irreducible component of $\Sigma_{\pi}$ Since the range of $f$ is contained in $g H$, this will show that the full $\mathbb{Z} \times G$ action for $\Sigma_{\pi}$ realizes the invariants $H$ and $g$. Let

$$
\begin{aligned}
Y & =\bigcup_{i=0}^{p-1} \sigma_{\pi}^{i}\left(\Sigma_{B} \times H\right) \\
& =\Sigma_{B} \times\left(\bigcup_{i=0}^{p-1}(g)^{i} H\right) \\
& =\Sigma_{B} \times G(g, H)
\end{aligned}
$$

where $G(g, H)$ is the group generated by $g$ and $H$. We will show that $Y$ is an irreducible component for $\Sigma_{\pi}$. Since $\sigma_{\pi}$ operates in the 2nd coordinate only by elements of $G(g, H)$, it immediately follows that $Y$ is an invariant set, and since $\Sigma_{B}$ is a direct factor of $Y, Y$ is a union of irreducible components. Thus it suffices to show that $\sigma_{\pi} \mid Y$ is transitive. In view of the irreducibility of $\Sigma_{B}$, it then suffices to show that for all pairs $h, h^{\prime} \in H$ there is a path in the graph of $\Sigma_{\pi}$ which starts at $(v, h)$ and ends at $\left(v, h^{\prime}\right)$. One does this by 'lifting' the cycle $\gamma_{i}$ where $i$ is defined by

$$
h_{i}=h^{\prime} h^{-1} \text {, }
$$

namely: if $\gamma_{i}=x_{1} \cdots x_{l}$ with $x_{1}=v$ and $x_{2}=s_{i}$ then the path

$$
\left(x_{1}, h\right)\left(x_{2}, f\left(x_{1} x_{2}\right) h\right) \cdots\left(x_{1}, f\left(x_{l} x_{1}\right) f\left(x_{l-1} x_{l}\right) \cdots f\left(x_{1} x_{2}\right) h\right)
$$

starts at $(v, h)$ and ends at $\left(v, h^{\prime}\right)$, as desired. (Recall that $l$ is a multiple of the order of $g$ in $S_{k}$.) So, $Y$ is an irreducible component. To see that $\Sigma_{B} \times H$ is a cyclic subset of $Y$ with period $p$, it simply suffices to find two cycles in the graph of $\Sigma_{\pi}$ which 
start in $\Sigma_{B} \times H$ and whose lengths are multiples of $p$ by a pair of relatively prime numbers. This is done by lifting the cycles $\alpha_{1}, \alpha_{2}$ to $\Sigma_{B} \times H$.

\section{Appendix}

Let $\Sigma_{B}$ be an irreducible SFT and $f_{1}, f_{2}: \Sigma_{B} \rightarrow S_{k}$ be locally constant functions. If $h$ is an almost continuous solution to the cohomology equation:

$$
f_{1}=\left(h \circ \sigma_{B}\right) \cdot f_{2} \cdot h^{-1} \text { a.e. }
$$

then $h$ is locally constant and in fact depends on at most as many coordinates as $f_{1}$ and $f_{2}$ do.

Proof. Assume for simplicity that $f_{1}$ and $f_{2}$ depend on only 1 coordinate. Since $h$ is almost continuous, there exists an element $\tau \in S_{k}$ and a cylinder set $C$ on which $h=\tau$ modulo a set of measure zero. By recoding, we may assume that $C$ is a 1-block cylinder $C=\left\{x: x_{0}=c\right\}$.

For $d \in L_{B}$ let $w=w_{0} \cdots w_{l}$ be a block such that $w_{0}=c$ and $w_{l}=d$. Let

$$
\alpha_{w}=\prod_{l-1}^{0} f_{2}\left(w_{i}\right) \text {. }
$$

(By this product, we mean $f_{2}\left(w_{l-1}\right) \cdots f_{2}\left(w_{0}\right)$.) Also let

$$
\bar{\alpha}_{w}=\prod_{l-1}^{0} f_{1}\left(w_{i}\right) .
$$

Define $\bar{h}: L_{B} \rightarrow S_{k}$ by

$$
\bar{h}(d)=\bar{\alpha}_{w} \tau \alpha_{w}^{-1} .
$$

We claim that $\bar{h}$ is well-defined (i.e. does not depend on the choice of block $w$ ). To see this, let $w=w_{0} \cdots w_{l}$ and $w^{1}=w_{0}^{1} \cdots w_{l}^{1}$ with

$$
w_{0}=w_{0}^{1}=c \text { and } w_{l}=w_{l^{\prime}}^{1}=d .
$$

Let $z=z_{1} z_{2} \cdots z_{k}$ be a path which begins with $d$ and ends with a predecessor of $c$. Then, $w z$ and $w^{1} z$ are both paths which begin with $c$ and end with a predecessor of $c$. Then, using the cohomology equation and the fact that $h$ is constant on $C$ $(\bmod 0)$, one sees that

$$
\bar{\beta} \bar{\alpha}_{w} \tau\left(\beta \alpha_{w}\right)^{-1}=\tau
$$

and

$$
\bar{\beta} \bar{\alpha}_{w^{1}} \tau\left(\beta \alpha_{w^{1}}\right)^{-1}=\tau
$$

where $\beta=\prod_{i=k}^{1} f_{2}\left(z_{i}\right)$ and $\bar{\beta}=\prod_{i=k}^{1} f_{1}\left(z_{i}\right)$. From this, one sees that

$$
\bar{\alpha}_{w} \tau \alpha_{w}^{-1}=\bar{\alpha}_{w^{1}} \tau \alpha_{w^{1}}^{-1} \text {. }
$$

So, $\bar{h}$ is well-defined. Then $\bar{h}$ defines a 1-block solution to the cohomology equation. (The reader can now verify that in fact $h=\bar{h} \bmod 0$ ).

B. Marcus was partially supported by NSF Grant MCS- 8301246 . 


\section{REFERENCES}

[1] R. Adler, B. Kitchens \& B. Marcus. Finite group actions on shifts of finite type. Ergod. Th. \& Dynam. Sys. 5 (1985), 1-25.

[2] R. Adler \& B. Marcus. Topological entropy and equivalence of dynamical systems. Mem. Amer. Math. Soc. 20 (1979), \#219.

[3] E. Coven \& M. Paul. Endomorphisms of irreducible subshifts of finite type. Math. Systems Theory 8 (1974), 167-175.

[4] E. Coven \& M. Paul. Finite procedures for sofic systems. Monats. Math. 83 (1977), 265-278.

[5] G. A. Hedlund. Endomorphisms and automorphisms of the shift dynamical system. Math. Systems Theory 3 (1969), 320-375.

[6] A. N. Livshits. Homology properties of $Y$-systems. Mathematical Notes of Academy of Sciences USSR 10 (1971), 751-763.

[7] B. Marcus. Factors and extensions of full shifts. Monats. Math. 88 (1979), 239-247.

[8] M. Nasu. Constant-to-one and onto global maps of homomorphisms between strongly connected graphs. Ergod. Th. \& Dynam. Sys. 3 (1983), 387-414.

[9] W. Parry \& S. Tuncel. Classification Problems in Ergodic Theory. London Math. Soc. Lecture Series \#67, Cambridge University Press, 1982.

[10] D. Rudolph. Counting the relatively finite factors of a Bernoulli shift. Israel J. Math. 30 (1978), 255-263. 